\title{
Strain rate sensitivity of the aluminium-magnesium-scandium alloy - Scalmalloy ${ }^{\circledR}$
}

\author{
Puneeth Jakkula ${ }^{1, *}$, Georg Ganzenmüller ${ }^{1,2, * *}$, Florian Gutmann ${ }^{2}$, and Stefan Hiermaier ${ }^{1,2}$ \\ ${ }^{1}$ Albert-Ludwigs-Universität Freiburg, Sustainable Systems Engineering - INATECH, Emmy-Noether- \\ Straße 2, 79110 Freiburg, Germany \\ ${ }^{2}$ Fraunhofer Institute for High-Speed Dynamics, Ernst-Mach-Institut - EMI, Ernst-Zermelo-Straße 4, \\ 79104 Freiburg, Germany
}

\begin{abstract}
This work investigates the strain rate sensitivity of the aluminiummagnesium-scandium alloy Scalmalloy, which is used extensively for additive manufacturing of lightweight structures. This high strength aluminium alloy combines very good weldability, machinability and mechanical strength: it can be heat-treated to reach nominal ultimate tensile strengths in excess of $500 \mathrm{MPa}$. We report tensile tests at strain rates ranging from $10^{-3} / \mathrm{s}$ to $10^{3} / \mathrm{s}$ at room temperature. It is well known that $\mathrm{Al}-\mathrm{Mg}$ alloys exhibit a negative strain rate dependency in combination with serrated flow caused by the Portevin-Le Chatelier effect, which describes the interaction of $\mathrm{Mg}$ solutes with dislocation propagations. In contrast, in Al-Sc alloys, the flow stress increases with increasing strain rate and displays positive strain rate dependency. Additionally, the presence of $\mathrm{Sc}$ in the form of $\mathrm{Al}_{3}-\mathrm{Sc}$ provides a fine-grained microstructure which allows higher tensile and fatigue strength. This research shows how these combined effects interact in the case of Scalmalloy, which contains both Mg and Sc. Tests are performed at quasi-static, intermediate and high strain rates with a servohydraulic testing machine and a Split-Hopkinson tension bar. Local specimen strain was performed using 2D Digital Image Correlation.
\end{abstract}

\section{Introduction}

Selective Laser Melting (SLM) of metal structures in Additive manufacturing (AM) has the ability to manufacture complex geometries without special tooling. This favours the production of lightweight, high strength materials which are increasingly utilised in various fields of automotive and aircraft industry [1-4]. The dynamic loading due to crash and impact are frequently encountered in these applications. It is therefore a necessity to know the strain-rate dependency of the material to quantify crashworthiness.

This work studies the Scandium modified Aluminium-Magnesium alloy AA5028 which has been developed and patented by APworks and sold under the trade name Scalmalloy. According to the manufacturer, it is based on the AA5xxx series Al-Mg alloys with an $\mathrm{Mg}$ content of 3.2 - 4.8 wt.- \% and Sc 0.02 - 0.04 wt.-\% [5]. The addition of Scandium improves the strength, weldability and corrosion resistance [6]. The yield stress of the as-built material is approximately $350 \mathrm{MPa}$ which can be increased by heat-treating to approximately

\footnotetext{
*e-mail: puneeth.jakkula@inatech.uni-freiburg.de

**e-mail: georg.ganzenmueller@inatech.uni-freiburg.de
} 
$550 \mathrm{MPa}$ [5]. These numbers, combined with the low mass density of $2.7 \mathrm{~g} / \mathrm{cm}^{3}$, make Scalmalloy an interesting candidate for lightweight crash-absorbing structures. Its strain-rate sensitivity, however, is difficult to predict. Al-Sc alloys exhibit positive strain rate sensitivity with increased yield stress at elevated strain rates [7]. In contrast, $\mathrm{Al}-\mathrm{Mg}$ alloys are known to exhibit negative strain rate sensitivity, at least up to moderate strain rates of $10^{3} / \mathrm{s}$ [8]. To answer the question on how these effects combine in Scalmalloy, we study its dynamic behaviour at four different strain rates ranging from $10^{-3} / \mathrm{s}$ to $1000 / \mathrm{s}$ using servo-hydraulic and split-Hopkinson testing methods. Both as-built (non-heat-treated) and heat-treated specimens are investigated, and the resulting data is analysed in terms of the strain-rate dependency of Ultimate Tensile Strength (UTS) and strain at failure.

\section{Material and methods}

\subsection{Scalmalloy}

A commercial Laser Beam Melting system (EOS M400) equipped with a $1 \mathrm{~kW}$ laser unit (YLR-series, Continuous Wave (CW)-laser, wavelength $1070 \mathrm{~nm}$ ) produced a total of 60 specimens from a single feedstock with dimensions of $\varnothing 20 \times 120 \mathrm{~mm}^{2}$. The specimens were printed in a 90 degree orientation to the build plate. Building platform heated to $40^{\circ} \mathrm{C}$ acted as the base to print the specimens in layers of $60 \mu \mathrm{m}$. According to APworks recommendation, half of the batch was heat treated for 4 hours at $325^{\circ} \mathrm{C}$. The specimen geometry shown in Figure 1 was CNC milled to dimensions. This geometry is chosen because it serves well with the constraints of our split-Hopkinson tension bar.

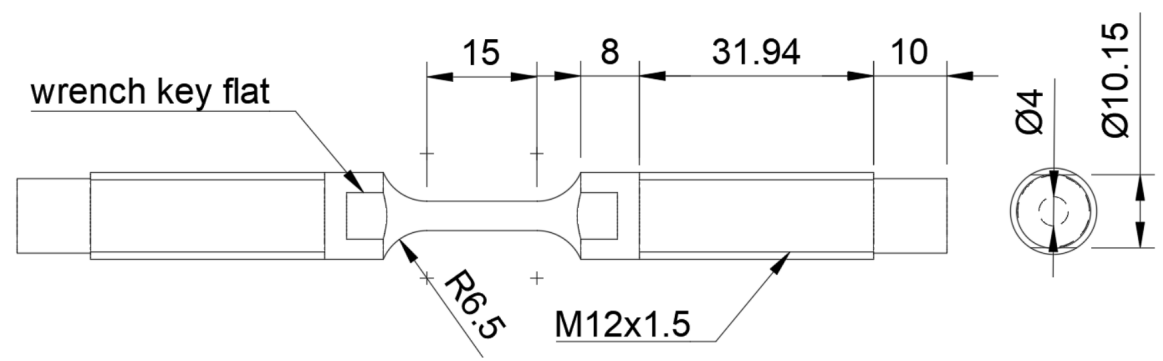

Figure 1. Specimen geometry with cylindrical gauge region of $\varnothing 4 \times 15$. All dimensions are in mm.

\subsection{Quasi static tensile testing}

The quasi static tensile tests were performed on the machine Instron 8801 system with $50 \mathrm{kN}$ load cell at room temperature. The cross-head velocities of the machine are chosen to achieve strain rates of $0.001 / \mathrm{s}, 0.1 / \mathrm{s}$ and $10 / \mathrm{s}$. Nominal specimen strain was calculated using 2D Digital Image Correlation (DIC) with the commercial code GOM correlate software, with a resolution of 1 pixel $=0.026 \mathrm{~mm}$. An airbrush gun is used to create a fine speckle pattern with an approximate speckle size of $0.1 \mathrm{~mm}$. High speed camera (Photron SAZ) with $1024 \times 1024$ resolution recorded the deformation images. A virtual extensometer (on 2D DIC images) was defined over the parallel gauge region to calculate the nominal strain. The nominal strain could be evaluated up to the point of failure. From prior experience, the accuracy of the nominal strain measurement is better than $2 \%$ of the indicated value. Additionally, we evaluated the local strain in the necking region. This, however, was only possible for small local strains, as DIC then failed to correctly track deformation field. The beginning of 
deviation between local strain and extensometer strain was used to define necking strain, i.e., the end of uniform elongation. In this work, we always address nominal (engineering) stress, i.e., the ratio of force over the initial value of the specimen's gauge length cross-section.

\subsection{Dynamic tensile testing}

A split-Hopkinson tension bar (SHTB) was used to perform dynamic testing at strain rate of $10^{3} / \mathrm{s}$. Figure 2 shows the configuration of SHTB apparatus. Compared to other SHTBs, this setup is optimized for low velocities, low forces and a long pulse duration of $1.2 \mathrm{~ms}$. Input and output bars are $16 \mathrm{~mm}$ diameter aluminium rods. The striker is a hollow aluminium tube of $40 \mathrm{~mm}$ outer diameter and $20 \mathrm{~mm}$ inner diameter. Strain gauge 1 on the input bar and strain gauge 2 on output bar measure the incident wave, $\varepsilon_{i n c}$, and transmitted wave, $\varepsilon_{\text {tra }}$ respectively. Force is measured via strain gauges on the bars, connected diagonally in a Wheatstone bridge circuit to eliminate bending information. The Wheatstone bridge circuit is driven in constant voltage mode and its output is increased by a factor of 100 using an amplifier. This signal is recorded by a data acquisition (DAQ) card operating at $10 \mathrm{MHz}$ and 16 bit resolution. The conversion factor from strain to force is established via a calibration procedure by placing a force censor between input and output bars, a static load is applied onto the bars. More details on the setup is described in [9]. Similar to that of quasi-static experiments the specimens are speckled with white and black spray paint with speckle resolution of 1 pixel $=0.067 \mathrm{~mm}$. A high speed camera (Photron SAZ, $640 \times 280$ pixels and 100,000 FPS) is employed for recording the deformation images. Specimen strain was computed from 2D DIC in the same manner as that of the low strain rate experiments.

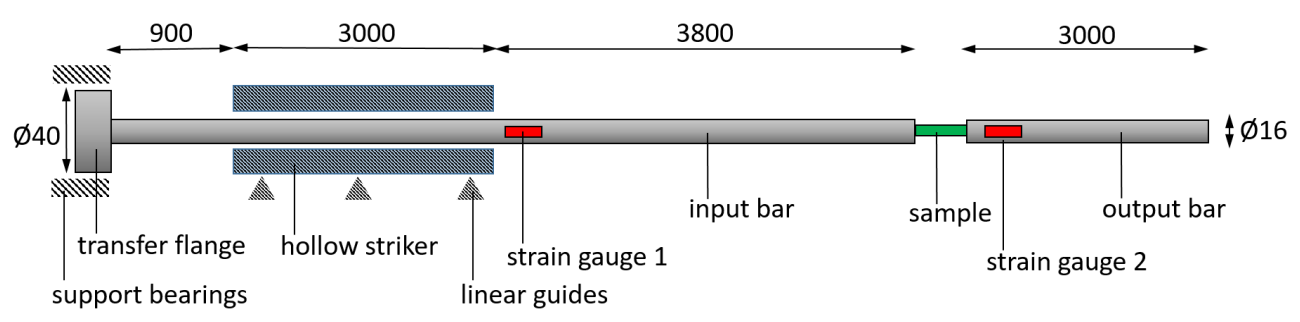

Figure 2. Sketch of the SHTB setup employed in this work. All dimensions are in mm.

\section{Results and discussion}

\subsection{Tensile tests results}

The nominal stress-strain curves are shown in Figure 3 for as-built and heat treated specimens. At strain rate $10^{-3} / \mathrm{s}$, obvious serrations in the curves are visible for as-built specimens. These are presumably due to the Portevin-Le-Chatelier (PLC) effect, which describes the locking of a moving dislocation due to solute $\mathrm{Mg}$ atoms [8]. At higher strain rates, this effect diminishes as the diffusion speed of solute $\mathrm{Mg}$ becomes slow compared to the dislocation speed.

The characteristic properties like ultimate tensile strength $\sigma_{\max }$, strain at failure $\varepsilon_{f}$, yield strength and necking strain are reported in Table 1 . Note that the value of the strain rate at yield is uncertain for the SHTB experiment as yielding occurs during the rise of the loading pulse. Our quasi-static data is in agreement with prior work on Scalmalloy [10], where an Ultimate Tensile Strength (UTS) of $334 \mathrm{MPa}$ and $540 \mathrm{MPa}$ was reported for as-built and heat-treated specimens, respectively. The failure behaviour is brittle for the heat-treated specimens, with almost no necking. Whereas, as-built specimens exhibit limited necking with diameter at the failure location of $75 \%$ to the initial value. 


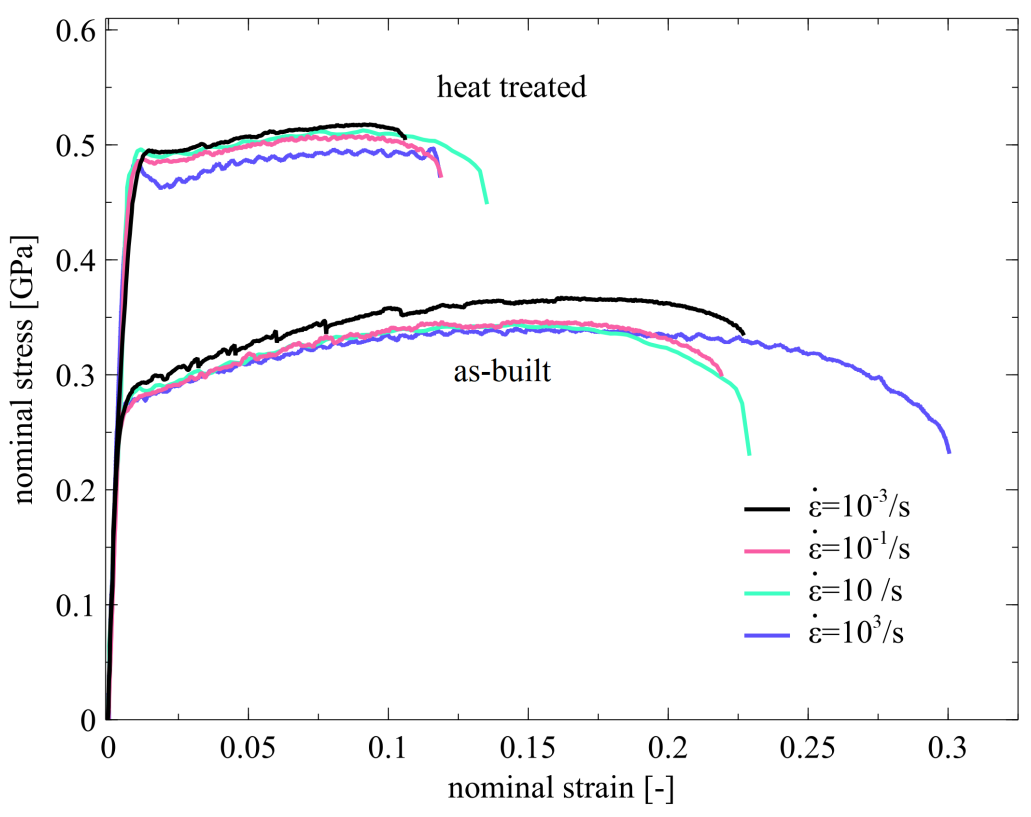

Figure 3. Scalmalloy nominal stress - nominal strain plots at different strain rates ranging from $10^{-3}$ to $10^{3} / \mathrm{s}$ for as-built (non heat-treated) and heat-treated specimens. Note that each curve is an average of 5 individual experiments.

Table 1. Results for ultimate tensile strength $\sigma_{\max }$, failure strain $\varepsilon_{\text {fail }}$, yield strength and necking strain at different strain rates considered in this work. Errors indicate one standard deviation.

\begin{tabular}{llllllllll}
\hline & \multirow{2}{*}{ strain rate $/ \mathrm{s}^{-1}$} & \multicolumn{2}{l}{ max. strength / MPa } & \multicolumn{2}{l}{ failure strain / \% } & \multicolumn{2}{l}{ yield strength / MPa } & \multicolumn{2}{l}{ necking strain / \% } \\
\hline as-built & 0.001 & 368.4 & \pm 1.1 & 23.2 & \pm 1.1 & 263.2 & \pm 4.2 & 17.8 & \pm 2.1 \\
& 0.1 & 348.5 & \pm 1.2 & 21.3 & \pm 1.1 & 260.8 & \pm 3.3 & 16.5 & \pm 0.9 \\
& 10 & 342.6 & \pm 0.9 & 23.0 & \pm 0.4 & 266.7 & \pm 1.2 & 15.9 & \pm 0.3 \\
& 1000 & 342.5 & \pm 1.6 & 29.5 & \pm 0.4 & 269.0 & \pm 7.1 & 23.1 & \pm 1.3 \\
heat-treated & 0.001 & 518.6 & \pm 0.5 & 11.2 & \pm 0.2 & 465.6 & \pm 1.2 & 10.3 & \pm 0.3 \\
& 0.1 & 508.1 & \pm 1.3 & 11.1 & \pm 0.5 & 470.7 & \pm 3.6 & 10.2 & \pm 0.6 \\
& 10 & 511.0 & \pm 1.5 & 12.8 & \pm 0.6 & 462.8 & \pm 1.8 & 10.8 & \pm 0.8 \\
& 1000 & 497.0 & \pm 3.0 & 15.0 & \pm 2.2 & 477.0 & \pm 3.9 & 14.9 & \pm 0.1 \\
\hline
\end{tabular}

\subsection{Strain rate dependency}

The characteristic quantities given in Table 1 are analysed to quantify the strain rate sensitivity. The maximum stress is well represented by a relationship proportional to the negative logarithm of the strain rate:

$$
\sigma_{\max }=\sigma_{0}+a \log (\dot{\varepsilon})
$$

The constant of proportionality for as-built is $a=-4.9 \pm 0.3 \mathrm{MPa}$, and for heat-treated is $a=-2.9 \pm 0.3 \mathrm{MPa}$. Uncertainty estimates are obtained from the residual error of the Levenberg-Marquardt algorithm used for data fitting. The dependence of failure strain on strain rate does not follow this simple Johnson-Cook type behaviour. Instead, a CowperSymonds relationship appears to fit the data better:

$$
\varepsilon_{\text {fail }}=\varepsilon_{\text {fail }, 0}\left(1+\alpha(\dot{\varepsilon} \times[\mathrm{s}])^{\beta}\right)
$$


For as-built (AB), we find $\varepsilon_{f a i l, 0}=0.223 \pm 0.005, \alpha=0.009 \pm 0.007$, and $\beta=0.51 \pm 0.10$. For heat-treated (HT), we find $\varepsilon_{\text {fail }, 0}=0.109 \pm 0.004, \alpha=0.009 \pm 0.007$, and $\beta=0.23 \pm 0.13$. In the strain rate interval $10^{-3}$ to $10 / \mathrm{s}$ both $(\mathrm{AB}$ and $\mathrm{HT})$ the strain at the end of uniform elongation (beginning of neck formation) and the failure strain are unaffected. However at $10^{3} / \mathrm{s}$, both failure strains increase. It can be said that this increase in ductility is the result of adiabatic heating. In conclusion, Scalmalloy exhibits a weak negative strain rate sensitivity for the UTS. The failure strain, however, increases along with the strain rate as shown in Figure 4.
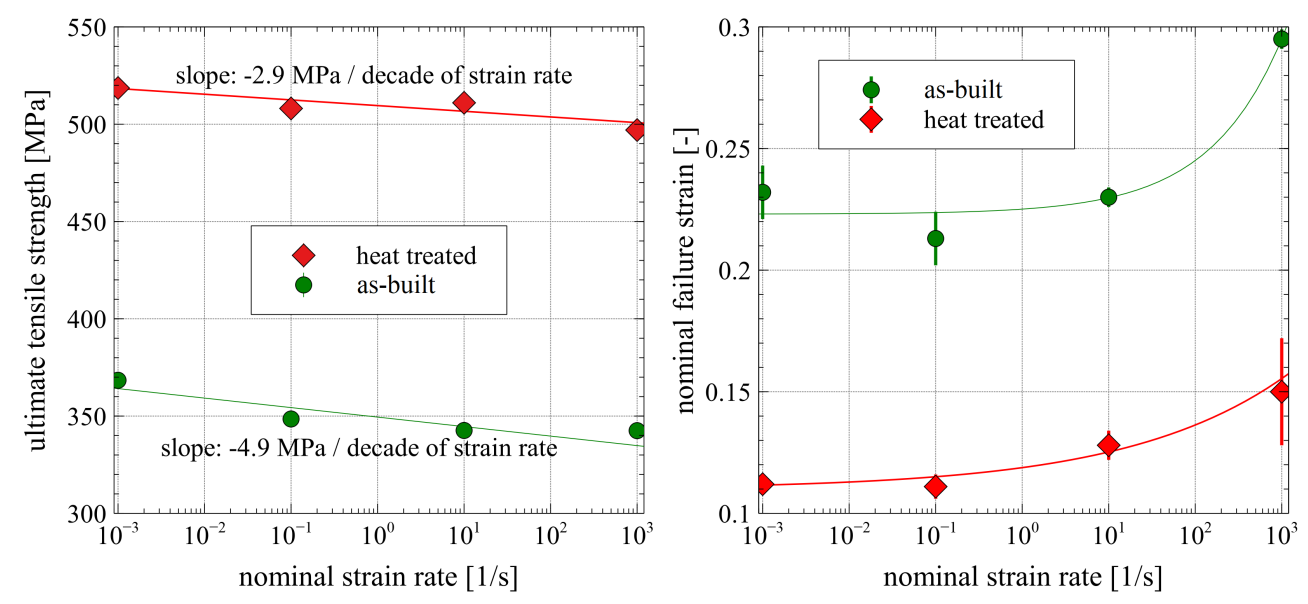

Figure 4. Analysis of the strain rate dependency of ultimate tensile strength and failure strain.

\section{Conclusion}

This work investigates the strain rate sensitivity of the Sc-Al-Mg alloy Scalmalloy used for additive manufacturing purposes. Tensile tests ranging from $10^{-3} / \mathrm{s}$ to $10^{3} / \mathrm{s}$ were performed with servo-hydraulic and split-Hopkinson methods. It was found that the Ultimate Tensile Strength (UTS) decreases weakly $(\approx 5 \mathrm{MPa} /$ decade of strain rate) with increasing strain rate, while the yield strength remains constant. Whereas, the failure strain increases along with rising strain rate, which is likely a consequence of adiabatic heating. In general, thermal softening leads to increase in ductility and reduction of strength. In Scalmalloy, UTS decreases slightly with strain rate, which might be the combined effect of both strain rate hardening as encountered with metals, and thermal softening. However, the fact that yield stress is unaffected by strain rate, c.f. Table 1, suggests that Scalmalloy shows no strain rate hardening at constant temperature, as adiabatic heating is not relevant at initial yield. The experiments reported here cannot separate these effects in practice, so we have to restrict our finding to an apparent weak decrease in UTS. Additional experiments that quantify the effects of varying temperature at constant strain rate are required to resolve this ambiguity. Further analysis of stress-strain data with Johnson-cook model can help in better understanding of the plastic deformation. Analysis of fracture surfaces with Scanning Electron Microscope (SEM) images shall explain the effects of heat-treatment on the microscope.

It is interesting to see how different strain rate effects of $\mathrm{Sc}$ and $\mathrm{Mg}$ combine in one aluminium alloy: the addition of $\mathrm{Mg}$ mainly leads to negative strain-rate sensitivity [8] at $\approx 10^{3} / \mathrm{s}$ of strain rate, while the addition of Sc incurs a pronounced positive strain rate sensitivity [11] for the UTS. For Scalmalloy, with alloying values of $4.4 \% \mathrm{Mg}$ and $0.73 \% \mathrm{Sc}$ by weight [10], these effects appear to mutually cancel out. We note that the observations 
made here only apply to strain rates of 1000 /s and below. One may speculate that, for even higher strain rates, a changeover from positive to negative strain rate exists, as is the case for the Al-Mg 5021 alloy [12].

\section{Acknowledgement}

We acknowledge funding from Carl-Zeiss Foundation, grant title Skalenübergreifende Charakterisierung robuster funktionaler Materialsysteme.

\section{References}

[1] Ozdemir, Z.; Hernandez-Nava, E.; Tyas, A.; Warren, J.A.; Fay, S.D.; Goodall, R.; Todd, I.; Askes, H. Energy absorption in lattice structures in dynamics: Experiments. International Journal of Impact Engineering 2016, 89, 49-61. doi:10.1016/j.ijimpeng.2015.10.007.

[2] Li, S.; Zhao, S.; Hou,W.; Teng, C.; Hao, Y.; Li, Y.; Yang, R.; Misra, R.D.K. Functionally Graded Ti-6Al-4V Meshes with High Strength and Energy Absorption. Advanced Engineering Materials 2016, 18, 34-38. doi:10.1002/adem.201500086.

[3] Tancogne-Dejean, T.; Spierings, A.B.; Mohr, D. Additively-manufactured metallic micro-lattice materials for high specific energy absorption under static and dynamic loading. Acta Materialia 2016, 116, 14-28. doi:10.1016/j.actamat.2016.05.054.

[4] Tsouknidas, A.; Pantazopoulos, M.; Katsoulis, I.; Fasnakis, D.; Maropoulos, S.; Michailidis, N. Impact absorption capacity of 3D-printed components fabricated by fused deposition modelling. Materials and Design 2016, 102, 41-44. doi:10.1016/j.matdes.2016.03.154.

[5] Vorel, M.; Hinsch, S.; Konopka, M.; Scheerer, M. AlMgSc alloy 5028 status of maturation 2017. p. 9 pages. Publisher: Proceedings of the 7th European Conference for Aeronautics and Space Sciences. Milano, Italy, 3-6 july (2017). doi:10.13009/EUCASS2017-633.

[6] Royset, J. Scandium In Aluminium Alloys: PhysicalMetallurgy, Properties And Applications. Metallurgical Science and Tecnology 2007, 25. Number: 2.

[7] Lee, W.S.; Chen, T.H. Dynamic Mechanical Response and Microstructural Evolution of High Strength Aluminum-Scandium (Al-Sc) Alloy. Mater. Trans. 2006, 47, 355-363. doi:10.2320/matertrans.47.355.

[8] Yamada, H.; Kami, T.; Mori, R.; Kudo, T.; Okada, M. Strain Rate Dependence of Material Strength in AA5xxx Series Aluminum Alloys and Evaluation of Their Constitutive Equation. Metals 2018, 8, 576. doi:10.3390/met8080576.

[9] Ganzenmüller, G.C.; Blaum, E.; Mohrmann, D.; Langhof, T.; Plappert, D.; Ledford, N.; Paul, H.; Hiermaier, S. A Simplified Design for a Split-Hopkinson Tension Bar with Long Pulse Duration. Procedia Engineering 2017, 197, 109-118. doi:10.1016/j.proeng.2017.08.087.

[10] Koutny, D.; Skulina, D.; Pante`lejev, L.; Paloušek, D.; Lenczowski, B.; Palm, F.; Nick, A. Processing of Al-Sc aluminum alloy using SLM technology. Procedia CIRP 2018, 74, 44-48. doi:10.1016/j.procir.2018.08.027.

[11] Lee, W.S.; Chen, T.H. Dynamic Deformation Behaviour and Microstructural Evolution of High-Strength Weldable Aluminum Scandium (Al-Sc) Alloy. Mater. Trans. 2008, 49, 1284-1293.doi:10.2320/matertrans.MRA2008032.

[12] Kami, T.; Yamada, H.; Ogasawara, N. Dynamic Behaviour of Al-Mg Aluminum Alloy at a Wide Range of Strain Rates. EPJ Web of Conferences 2018, 183, 02028. doi:10.1051/epjconf/201818302028. 